\title{
Excess Volumes of 1,2,4-Trichlorobenzene + Methyl Ethyl Ketone + 1-Alkanols at $303.15 \mathrm{~K}$
}

\author{
Jalari R. Sekar and Puligundla R. Naidu* \\ Department of Chemistry, Sri Venkateswara University College of Arts and Sciences, Tirupati 517 502, India
}

William E. Acree, Jr.

Department of Chemistry, University of North Texas, Denton, Texas 76203-5068

Excess volumes for 1,2,4-trichlorobenzene + methyl ethyl ketone + 1-propanol, + 1-butanol, + 1-pentanol, and +1 -hexanol were measured at $303.15 \mathrm{~K}$. Excess volumes are negative over the entire range of composition in the four mixtures. The results were compared with those predicted by empirical equations proposed by Hwang et al. and Redlich-Kister.

\section{Introduction}

Hwang et al. (1) have recently developed a semiempirical equation that includes contributions from both two-body and three-body interactions. The relation has been used to predict ternary Gibbs free energies of several aqueous-organic systems. Acree and Naidu (3) extended the relation to excess volumes of six ternary mixtures and noted that the equation gives a satisfactory estimate of the excess property. Recently Raja Sekar, Naidu, and Acree (4) and also Siva Kumar, Naidu, and Acree (5) analyzed excess volumes of seven ternary mixtures on the basis of the equation and concluded that it gives a rough estimate of the excess volume. We report here new experimental excess volumes for the binary mixture 1,2,4trichlorobenzene + methyl ethyl ketone, and for four ternary mixtures containing 1,2,4-trichlorobenzene, methyl ethyl ketone, and four 1-alkanols (1-propanol, 1-butanol, 1-pentanol, and 1-hexanol). The results were used to check the capability of the predictive expressions developed by Hwang et al. (1) and Redlich-Kister (2).

\section{Purification of Materials}

All the chemicals used were of analytical grade. 1,2,4Trichlorobenzene was purified by the method described by Vijayalakshmi and Naidu (11). Methyl ethyl ketone and 1-alkanols were purified by the methods described by Reddick and Bunger (7). 1,2,4-Trichlorobenzene (Merck) was purified by repeated fractional distillation and stored in the dark with the vapor phase in contact with anhydrous magnesium perchlorate. The methyl ethyl ketone and 1-alkanols were purified as described in an earlier paper (4). The purity of the sample was checked by comparing the measured densities of the compounds with those reported in the literature $(8,11)$ and single sharp GLC peaks. Densities were determined with a bicapillary-type pycnometer (9), which offers an accuracy of 2 parts in $10^{5}$. The measured densities and those reported in the literature are given in Table 1.

\section{Experimental Procedure}

Excess volumes were measured with the dilatometer described by Naidu and Naidu (6). The mixing cell contained three bulbs of different capacities. Mercury was used at the

* To whom correspondence should be addressed.

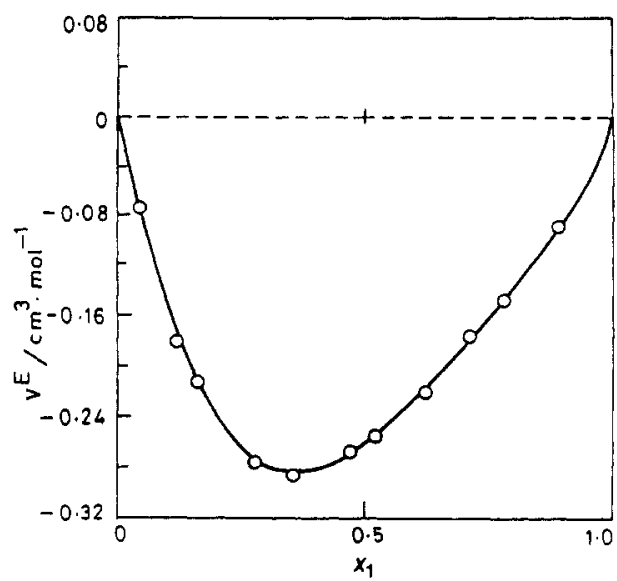

Figure 1. Excess volume $\left(V^{\mathbb{E}}\right)$ plotted against the mole fraction of 1,2,4-trichlorobenzene $\left(x_{1}\right)$ with methyl ethyl ketone at $303.15 \mathrm{~K}$.

Table 1. Densities ( $\rho$ ) of Pure Components at 303.15 K

\begin{tabular}{|c|c|c|}
\hline \multirow[b]{2}{*}{ component } & \multicolumn{2}{|c|}{$\rho /\left(\mathrm{g} \cdot \mathrm{cm}^{-3}\right)$} \\
\hline & lit. & present study \\
\hline 1,2,4-trichlorobenzene & $1.44215(11)$ & 1.44213 \\
\hline methyl ethyl ketone & $0.79452(8)$ & 0.79457 \\
\hline 1-propanol & $0.79600(8)$ & 0.79602 \\
\hline 1-butanol & $0.80206(8)$ & 0.80203 \\
\hline 1-pentanol & $0.80764(8)$ & 0.80764 \\
\hline 1-hexanol & $0.81201(8)$ & 0.81205 \\
\hline
\end{tabular}

Table 2. Excess Volumes for 1,2,4-Trichlorobenzene (1) + Methyl Ethyl Ketone (2) at $303.15 \mathrm{~K}$

\begin{tabular}{|c|c|c|c|}
\hline$x_{1}$ & $\begin{array}{c}\mathrm{VE} / \\
\left(\mathrm{cm}^{3}\right. \\
\left.\mathrm{mol}^{-1}\right)\end{array}$ & $x_{1}$ & $\begin{array}{c}\mathrm{VE} / \\
\left(\mathrm{cm}^{3}\right. \\
\left.\mathrm{mol}^{-1}\right)\end{array}$ \\
\hline 0.0365 & -0.072 & 0.5184 & -0.255 \\
\hline 0.1200 & -0.179 & 0.6236 & -0.218 \\
\hline 0.1551 & -0.212 & 0.7147 & -0.177 \\
\hline 0.2813 & -0.276 & 0.7783 & -0.148 \\
\hline 0.3637 & -0.283 & 0.8942 & -0.089 \\
\hline 0.4682 & -0.267 & & \\
\hline
\end{tabular}

bottom to separate the three components. One of the three bulbs was fitted with a capillary, and the other two were fitted with ground-glass stoppers. Four dilatometers of the aforementioned type were used to cover the entire mole fraction range. All measurements were made at constant 
Table 3. Binary Parameters and the Standard Deviation Obtained by the Method of Least Squares Using the

Redlich-Kister Equation (4)

\begin{tabular}{|c|c|c|c|c|}
\hline system & $\begin{array}{l}a_{0}^{(i j)} / \\
\left(\mathrm{cm}^{3} .\right. \\
\left.\mathrm{mol}^{-1}\right)\end{array}$ & $\begin{array}{c}a_{1}^{(i j)} / \\
\left(\mathrm{cm}^{3} .\right. \\
\left.\mathrm{mol}^{-1}\right)\end{array}$ & $\begin{array}{l}a_{2}^{(i j)} / \\
\left(\mathrm{cm}^{3} .\right. \\
\left.\mathrm{mol}^{-1}\right)\end{array}$ & $\begin{array}{c}\sigma\left(V^{\mathbf{E}}\right) / \\
\left(\mathrm{cm}^{3} .\right. \\
\left.\mathrm{mol}^{-1}\right)\end{array}$ \\
\hline $\begin{array}{l}\text { 1,2,4-trichlorobenzene (1) + } \\
\text { methyl ethyl ketone (2) }\end{array}$ & -1.0203 & 0.5455 & -0.5408 & 0.005 \\
\hline $\begin{array}{l}\text { 1,2,4-trichlorobenzene }(1)+ \\
1 \text {-propanol }(3)^{a}\end{array}$ & -0.1425 & 1.0004 & -0.3142 & 0.004 \\
\hline $\begin{array}{l}\text { methyl ethyl ketone }(2)+ \\
1 \text {-propanol }(3)^{b}\end{array}$ & -0.1709 & 0.0122 & 0.0118 & 0.001 \\
\hline $\begin{array}{l}\text { 1,2,4-trichlorobenzene }(1)+ \\
1 \text {-butanol }(3)^{c}\end{array}$ & -0.3080 & 0.9261 & -0.0972 & 0.005 \\
\hline $\begin{array}{l}\text { methyl ethyl ketone }(2)+ \\
1 \text {-butanol }(3)^{b}\end{array}$ & 0.0598 & 0.0283 & 0.0156 & 0.002 \\
\hline $\begin{array}{l}\text { 1,2,4-trichlorobenzene }(1)+ \\
1 \text {-pentanol }(3)^{c}\end{array}$ & -0.1646 & 0.8926 & -0.0515 & 0.004 \\
\hline $\begin{array}{l}\text { methyl ethyl ketone }(2)+ \\
1 \text {-pentanol }(3)^{b}\end{array}$ & 0.1615 & 0.0662 & -0.0960 & 0.004 \\
\hline $\begin{array}{l}\text { 1,2,4-trichlorobenzene (1) }+ \\
\text { 1-hexanol (3) }\end{array}$ & -0.1711 & 0.6949 & 0.3431 & 0.006 \\
\hline $\begin{array}{l}{ }^{b} \text { methyl ethyl ketone }(2)+ \\
1 \text {-hexanol (3) }\end{array}$ & 0.3133 & 0.0051 & 0.1721 & 0.002 \\
\hline
\end{tabular}

Table 4. Binary Parameters and the Standard Deviation Obtained by Using the Hwang et al. equation (2)

\begin{tabular}{|c|c|c|c|c|}
\hline system & $\begin{array}{l}V_{0}^{(i j)} / \\
\left(\mathrm{cm}^{3} .\right. \\
\left.\mathrm{mol}^{-1}\right)\end{array}$ & $\begin{array}{l}V_{1}^{(i)} / \\
\left(\mathrm{cm}^{3} .\right. \\
\left.\mathrm{mol}^{-1}\right)\end{array}$ & $\begin{array}{l}V_{2}^{(i j)} / \\
\left(\mathrm{cm}^{3} .\right. \\
\left.\mathrm{mol}^{-1}\right)\end{array}$ & $\begin{array}{c}\sigma\left(V^{E}\right) / \\
\left(\mathrm{cm}^{3}\right. \\
\left.\mathrm{mol}^{-1}\right)\end{array}$ \\
\hline $\begin{array}{l}\text { 1,2,4-trichlorobenzene (1) + } \\
\text { methyl ethyl ketone (2) }\end{array}$ & -0.8399 & 0.0726 & -1.3711 & 0.006 \\
\hline $\begin{array}{l}\text { 1,2,4-trichlorobenzene (1) + } \\
\text { 1-propanol (3) }\end{array}$ & -0.0379 & 0.7735 & -1.6091 & 0.004 \\
\hline $\begin{array}{l}\text { methyl ethyl ketone }(2)+ \\
\text { 1-propanol (3) }\end{array}$ & -0.1744 & 0.0282 & 0.0007 & 0.001 \\
\hline $\begin{array}{l}\text { 1,2,4-trichlorobenzene }(1)+ \\
\quad \text {-butanol (3) }\end{array}$ & -0.2757 & 0.9735 & -1.2322 & 0.008 \\
\hline $\begin{array}{l}\text { methyl ethyl ketone (2) + } \\
\text { 1-butanol (3) }\end{array}$ & 0.0552 & 0.0530 & -0.0147 & 0.002 \\
\hline $\begin{array}{l}\text { 1,2,4-trichlorobenzene }(1)+ \\
\text { 1-pentanol (3) }\end{array}$ & -0.1478 & 0.9949 & -1.1308 & 0.007 \\
\hline $\begin{array}{l}\text { methyl ethyl ketone (2) + } \\
\text { 1-pentanol (3) }\end{array}$ & 0.1891 & -0.0359 & -0.1969 & 0.011 \\
\hline $\begin{array}{l}\text { 1,2,4-trichlorobenzene }(1)+ \\
\text { 1-hexanol (3) }\end{array}$ & -0.2859 & 1.2863 & -0.3688 & 0.006 \\
\hline $\begin{array}{l}\text { methyl ethyl ketone }(2)+ \\
\text { 1-hexanol (3) }\end{array}$ & 0.2559 & 0.2342 & 0.2241 & 0.020 \\
\hline
\end{tabular}

temperature in a thermostat maintained to $\pm 0.01 \mathrm{~K}$. The measured $V^{E}$ values were accurate to $\pm 0.003 \mathrm{~cm}^{3} \cdot \mathrm{mol}^{-1}$.

\section{Results and Discussion}

Hwang et al. (1) developed the relation

$$
A^{\mathrm{E}(i j)}=G^{\mathrm{E}(i j)}=x_{i} x_{j}\left(V_{0}^{(i j)}+V_{1}^{(i j)} x_{i}^{3}+V_{2}^{(i j)} x_{j}{ }^{3}\right)
$$

where $A^{\mathrm{E}(i j)}$ or $G^{\mathrm{E}(i j)}$ represents an excess thermodynamic property. $x_{i}$ and $x_{j}$ denote the mole fractions of components $i$ and $j . V_{0}, V_{1}$, and $V_{2}$ are the constants for binary mixtures, taking into account two-body and three-body interactions.

Acree and Naidu (3) adopted the equation for excess volume in the following form:

$$
V^{\mathrm{E}(i j)}=x_{i} x_{j}\left(V_{0}^{(i j)}+V_{1}^{(i j)} x_{i}^{3}+V_{2}^{(i j)} x_{j}^{3}\right)
$$

These researchers used the predictive expression to compute the ternary data from those of the constituent binaries in the following form:
Table 5. Experimental and Predicted Excess Molar Volumes of Ternary Mixtures for 1,2,4-Trichlorobenzene

\begin{tabular}{|c|c|c|c|c|}
\hline \multirow[b]{2}{*}{$x_{1}$} & \multirow[b]{2}{*}{$x_{2}$} & \multicolumn{3}{|c|}{$V^{\mathrm{E}}(123) /\left(\mathrm{cm}^{3} \cdot \mathrm{mol}^{-1}\right)$} \\
\hline & & $\exp$ & $\begin{array}{l}\text { Hwang } \\
\text { et al. }\end{array}$ & $\begin{array}{l}\text { Redlich- } \\
\text { Kister }\end{array}$ \\
\hline \multicolumn{5}{|c|}{$\begin{array}{c}\text { 1,2,4-Trichlorobenzene (1) + } \\
\text { Methyl Ethyl Ketone (2) + 1-Propanol (3) }\end{array}$} \\
\hline 0.1536 & 0.0710 & -0.172 & -0.112 & -0.126 \\
\hline 0.1113 & 0.2185 & -0.145 & -0.085 & -0.112 \\
\hline 0.1228 & 0.2687 & -0.154 & -0.087 & -0.118 \\
\hline 0.1229 & 0.4250 & -0.149 & -0.093 & -0.125 \\
\hline 0.1298 & 0.5134 & -0.156 & -0.105 & -0.136 \\
\hline 0.1570 & 0.6251 & -0.197 & -0.140 & -0.166 \\
\hline 0.1220 & 0.7300 & -0.180 & -0.141 & -0.159 \\
\hline 0.1056 & 0.8109 & -0.167 & -0.146 & -0.155 \\
\hline 0.0722 & 0.8784 & -0.131 & -0.119 & -0.122 \\
\hline \multicolumn{5}{|c|}{$\begin{array}{l}\text { 1,2,4-Trichlorobenzene (1) + } \\
\text { Methyl Ethyl Ketone (2) + 1-Butanol (3) }\end{array}$} \\
\hline 0.1746 & 0.1249 & -0.145 & -0.099 & -0.118 \\
\hline 0.1285 & 0.2160 & -0.119 & -0.069 & -0.092 \\
\hline 0.1412 & 0.2928 & -0.125 & -0.067 & -0.095 \\
\hline 0.1368 & 0.3745 & -0.122 & -0.064 & -0.093 \\
\hline 0.1500 & 0.4519 & -0.135 & -0.076 & -0.105 \\
\hline 0.1267 & 0.5476 & -0.128 & -0.076 & -0.102 \\
\hline 0.1450 & 0.6031 & -0.148 & -0.100 & -0.125 \\
\hline 0.1821 & 0.7096 & -0.201 & -0.172 & -0.187 \\
\hline 0.0997 & 0.7942 & -0.134 & -0.117 & -0.127 \\
\hline 0.0858 & 0.8630 & -0.132 & -0.125 & -0.128 \\
\hline \multicolumn{5}{|c|}{$\begin{array}{l}\text { 1,2,4-Trichlorobenzene (1) + } \\
\text { Methyl Ethyl Ketone (2) + 1-Pentanol (3) }\end{array}$} \\
\hline 0.2012 & 0.1079 & -0.159 & -0.080 & -0.100 \\
\hline 0.1617 & 0.2060 & -0.133 & -0.054 & -0.081 \\
\hline 0.1683 & 0.2526 & -0.128 & -0.050 & -0.079 \\
\hline 0.1571 & 0.2954 & -0.117 & -0.044 & -0.074 \\
\hline 0.1490 & 0.4079 & -0.118 & -0.042 & -0.073 \\
\hline 0.1476 & 0.4804 & -0.130 & -0.050 & -0.080 \\
\hline 0.1409 & 0.5775 & -0.145 & -0.068 & -0.094 \\
\hline 0.1393 & 0.6750 & -0.172 & -0.100 & -0.122 \\
\hline 0.0988 & 0.7579 & -0.132 & -0.091 & -0.106 \\
\hline 0.0795 & 0.8706 & -0.132 & -0.114 & -0.118 \\
\hline \multicolumn{5}{|c|}{$\begin{array}{c}\text { 1,2,4-Trichlorobenzene (1) }+ \\
\text { Methyl Ethyl Ketone (2) + 1-Hexanol (3) }\end{array}$} \\
\hline 0.2172 & 0.1113 & -0.106 & -0.052 & -0.057 \\
\hline 0.1709 & 0.2185 & -0.096 & -0.029 & -0.037 \\
\hline 0.1617 & 0.3337 & -0.104 & -0.026 & -0.038 \\
\hline 0.1550 & 0.4541 & -0.109 & -0.035 & -0.050 \\
\hline 0.1477 & 0.5398 & -0.110 & -0.047 & -0.063 \\
\hline 0.1491 & 0.6342 & -0.123 & -0.078 & -0.093 \\
\hline 0.1533 & 0.7379 & -0.160 & -0.134 & -0.144 \\
\hline 0.1186 & 0.8180 & -0.151 & -0.137 & -0.141 \\
\hline 0.0755 & 0.8714 & -0.108 & -0.097 & -0.099 \\
\hline \multicolumn{5}{|c|}{$\begin{array}{l}V^{\mathrm{E}(123)}=x_{1} x_{2}\left(V_{0}^{(12)}+V_{1}^{(12)} x_{1}^{3}+V_{2}^{(12)} x_{2}^{3}\right)+ \\
\quad x_{1} x_{3}\left(V_{0}^{(13)}+V_{1}^{(13)} x_{1}^{3}+V_{3}^{(13)} x_{3}^{3}\right)+x_{2} x_{3}\left(V_{0}^{(23)}+\right.\end{array}$} \\
\hline
\end{tabular}
(1) + Methyl Ethyl Ketone (2) + 1-Alkanols (3) at 303.15 K

Redlich and Kister (2) (R-K) proposed the equation for binary excess volume data:

$$
V^{\mathrm{E}(i j)}=x_{i} x_{j}\left[a_{0}{ }^{(i j)}+a_{1}{ }^{(i j)}\left(x_{i}-x_{j}\right)+a_{2}^{(i j)}\left(x_{i}-x_{j}\right)^{2}\right]
$$

where $x_{i}$ and $x_{j}$ denote the mole fractions of components $i$ and $j . a_{0}, a_{1}$, and $a_{2}$ are the constants. The equation, when extended to a ternary mixture, takes the form

$$
\begin{gathered}
V^{\mathrm{E}(123)}=x_{1} x_{2}\left[a_{0}{ }^{(12)}+a_{1}{ }^{(12)}\left(x_{1}-x_{2}\right)+a_{2}{ }^{(12)}\left(x_{1}-x_{2}\right)^{2}\right]+ \\
x_{1} x_{3}\left[a_{0}{ }^{(13)}+a_{1}{ }^{(13)}\left(x_{1}-x_{3}\right)+a_{2}{ }^{(13)}\left(x_{1}-x_{3}\right)^{2}\right]+ \\
x_{2} x_{3}\left[a_{0}{ }^{(23)}+a_{1}{ }^{(23)}\left(x_{2}-x_{3}\right)+a_{2}{ }^{(23)}\left(x_{2}-x_{3}\right)^{2}\right]
\end{gathered}
$$

Excess volumes for the four ternary mixtures from eqs 3 and 
Table 6. Values of Ternary Constants $A, B$, and $C$ and $\sigma$ at $303.15 \mathrm{~K}$

\begin{tabular}{ccccc}
\hline \multicolumn{1}{c}{ system } & $\begin{array}{c}\mathrm{A} / \\
\left(\mathrm{cm}^{3} .\right. \\
\left.\mathrm{mol}^{-1}\right)\end{array}$ & $\begin{array}{c}\mathrm{B} / \\
\left(\mathrm{cm}^{3} .\right. \\
\left.\mathrm{mol}^{-1}\right)\end{array}$ & $\begin{array}{c}\mathrm{C} / \\
\left(\mathrm{cm}^{3} .\right. \\
\left.\mathrm{mol}^{-1}\right)\end{array}$ & $\begin{array}{c}\sigma / \\
\left(\mathrm{cm}^{3} .\right. \\
\left.\mathrm{mol}^{-1}\right)\end{array}$ \\
\hline $\begin{array}{l}\text { 1,2,4-trichlorobenzene (1)+ } \\
\text { methyl ethyl ketone (2) + }\end{array}$ & -1.0540 & 8.1834 & -284.1176 & 0.004 \\
$\begin{array}{l}\text { 1-propanol (3) } \\
\text { 1,2,4-trichlorobenzene (1) + } \\
\text { methyl ethyl ketone (2) }+\end{array}$ & -0.9822 & 3.9949 & -63.7243 & 0.004 \\
$\begin{array}{l}\text { 1-butanol (3) } \\
\text { 1,2,4-trichlorobenzene (1) }+ \\
\text { methyl ethyl ketone (2) }+\end{array}$ & -1.6030 & -7.6615 & -250.5834 & 0.005 \\
$\begin{array}{l}\text { 1-pentanol (3) } \\
\text { 1,2,4-trichlorobenzene (1) }+ \\
\text { methyl ethyl ketone (2) }+ \\
\text { 1-hexanol (3) }\end{array}$ & -2.2880 & 6.8913 & 13.5473 & 0.004 \\
& & & & \\
\end{tabular}

5 are included in Table 5. Binary parameters appearing in the $\mathrm{R}-\mathrm{K}$ equation for 1,2,4-trichlorobenzene with 1-alkanols and methyl ethyl ketone with 1-alkanols were taken from the literature $(10-12)$. The parameters for the system 1,2,4trichlorobenzene with methyl ethyl ketone were computed from the $V^{\mathrm{E}}$ data measured in the present investigation. These $V^{\mathrm{E}}$ results are included in Table 2 and are also graphically represented in Figure 1. All the binary parameters obtained by using eq 4 relating to the constituent binary mixtures are included in Table 3.

The coefficients in eq 2 were computed from the smoothed binary experimental results at mole fractions $x_{i}=0.2,0.5$, and 0.8 . In the computation of the coefficients, values of $V^{\mathrm{E}}$ at $x=0.2,0.5$, and 0.8 were estimated using the RedlichKister equation (4)

$$
V^{E(i j)}=x_{i} x_{j}\left[a_{0}+a_{1}\left(x_{i}-x_{j}\right)+a_{2}\left(x_{i}-x_{j}\right)^{2}\right]
$$

and the values of binary parameters are given in Table 3 . The estimated values of $V^{E}$ were then substituted in the Hwang et al. equation (2)

$$
V^{\mathrm{E}(i j)}=x_{i} x_{j}\left(V_{0}+V_{1} x_{i}^{3}+V_{2} x_{j}^{3}\right)
$$

at $x=0.2,0.5$, and 0.8 . The resulting three equations were then solved to give the values of $V_{0}, V_{1}$, and $V_{2}$. The values of the coefficients relating to the constituent binary mixtures are included in Table 4.
The experimental $V^{E}$ results for the ternary mixtures and those predicted by the Hwang et al. and Redlich-Kister models are given in columns $3-5$ of Table 5 , respectively.

The dependence of the experimental ternary excess volume, $V_{(\exp )}^{\mathrm{E}(123)}$, on the composition is expressed by the polynomial

$$
\begin{aligned}
V_{(\exp )}^{\mathrm{E}(123)}=V_{(\mathrm{b})}^{\mathrm{E}(123)}+x_{1} x_{2} x_{3}\left[A+B x_{1}\left(x_{2}-x_{3}\right)+\right. \\
\left.C x_{1}{ }^{2}\left(x_{2}-x_{3}\right)^{2}\right]
\end{aligned}
$$

where $V_{(b)}^{\mathbb{E}(123)}=V^{\mathrm{E}(12)}+V^{\mathrm{E}(13)}+V^{\mathrm{E}(23)}$ and $x_{1}, x_{2}$, and $x_{3}$ are mole fractions of 1,2,4-trichlorobenzene, methyl ethyl ketone, and an alcohol. $A, B$, and $C$ are ternary constants, and their values, computed by the least-squares method, are given in Table 6.

An examination of the results in Table 5 shows that the predictive expressions give $V^{\mathbb{E}}$ data that are algebraically larger than the experimental data. The difference between the two sets far exceed the experimental error. Hence, it is concluded that predictive expressions are incapable of estimating the excess property accurately.

\section{Literature Cited}

(1) Hwang, C. A.; Holste, J. C.; Hall, K. R.; Mansori, G. A. Fluid Phase Equilib. 1991, 62, 173.

(2) Redlich, O.; Kister, A. T. Ind. Eng. Chem. 1948, 40, 345.

(3) Acree, W. E., Jr.; Naidu, P. R. Phys. Chem. Liq., in press.

(4) Sekar, J. R.; Naidu, P. R.; Acree, W. E., Jr. J. Chem. Eng. Data 1993, $38,167-169$

(5) Sivakumar, K.; Naidu, P. R.; Acree, W. E., Jr. J. Chem. Eng. Dato $1994,39,2-4$.

(6) Naidu, G. R.; Naidu, P. R. J. Chem. Eng. Data 1981, 26, 197.

(7) Riddick, J. A.; Bunger, W. B. Techniques of Chemistry, 3rd ed.; Wiley Interscience: New York, 1970.

(8) Timmermans, J. Physico-Chemical constants of pure organic compounds; Elsevier: New York, 1950.

(9) Rao, M. V. P. Ph.D. Thesis, Sri Venkateswara University, India, 1974.

(10) Chandrakumar, V.; Naidu, P. R. Phys. Chem. Liq., in press.

(11) Vijayalakshmi, T. S.; Naidu, P. R. J. Chem. Eng. Data 1992, 37, 368-369.

(12) Reddy, K. S.; Naidu, P. R. Can. J. Chem. 1977, 55, 76.

Received for review September 20, 1993. Revised January 28, 1994. Accepted March 2, 1994.

- Abstract published in Advance ACS Abstracts, April 15, 1994. 